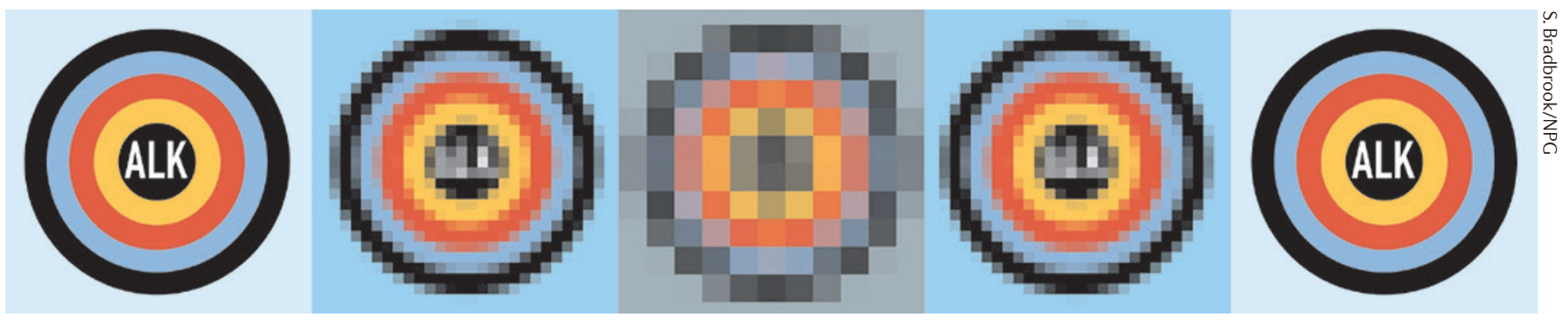

LUNG CANCER

\title{
Resolving resistance to ALK-targeted therapy
}

Disease resistance is one of the biggest challenges in achieving cure or long-term remission from cancer. In patients who respond and then relapse, treatment with moreselective, next-generation inhibitors is used to induce a response. Resistance to crizotinib is mediated by a variety of mechanisms, which sometimes involve secondary mutations within the ALK catalytic domain that render it resistant to treatment. Lorlatinib is a new-generation ALK inhibitor in early phase clinical testing.

Alice Shaw and co-authors have published a case study of a 52-year-old woman with metastatic $A L K$-rearranged non-small-cell lung cancer (NSCLC) who was treated with multiple therapies, which included crizotinib, ceritinib, and lorlatinib. When the cancer relapsed on lorlatinib, the patient deteriorated rapidly and developed liver failure. Molecular assessment of the lorlatinib-resistant tumour specimen enabled the identification of a novel ALK aberration (ALK L1198F) that resulted in this resistance. Remarkably, while this mutation caused resistance to lorlatinib, it paradoxically enhanced sensitivity to crizotinib. Consistent with these findings, the patient improved almost immediately when she was retreated with crizotinib. Her disease-related symptoms and liver failure completely resolved, and she was able to resume her normal life.

For oncogene-addicted cancers, such as $A L K$-rearranged NSCLC patients are typically treated in a linear fashion with first-generation targeted therapies, such as crizotinib, and upon relapse, are then switched to receive an increasingly potent and selective targeted therapy. As Shaw highlights, "we would usually never consider returning to an earliergeneration drug because there is typically cross resistance. This case proves that that notion may be incorrect and highlights the critical importance of identifying each patient's underlying mechanism of resistance, as the information obtained may be crucial to finding the most-effective treatment options."

In terms of future work, Shaw and her team plan to study other lung cancers that have become resistant to lorlatinib and other next-generation ALK inhibitors to determine the incidence of the ALK L1198F mutation across different resistant cancers. "We are also analysing circulating tumour DNA from patients to monitor in real-time the emergence of ALK resistance mutations, which include ALK L1198F."

Lisa Hutchinson

ORIGINAL ARTICLE Shaw, A. T. et al.

Resensitization to crizotinib by the lorlatinib ALK resistance mutation L1198F. N. Engl.J. Med. 374, 54-61 (2016) 